\title{
VIGILÂNCIA EM SAÚDE DO TRABALHADOR NO BRASIL: CONCEITO E DESAFIOS
}

\section{HEALTH SURVEILLANCE OF WORKERS IN BRAZIL: CONCEPT AND CHALLENGES}

Geyse Chrystine Pereira Souza Fernandes ${ }^{1}$

\section{Resumo}

A Vigilância em Saúde do Trabalhador atua nas relações do processo de trabalho com a saúde e é um meio fundamental para promover a saúde dos trabalhadores e a prevenção de acidentes e de doenças relacionadas ao trabalho; sendo imprescindível refletir sobre conceitos e políticas voltadas para a saúde do trabalhador. Esse artigo tem uma abordagem qualitativa do tipo bibliográfica descritiva, assim esta revisão tem o objetivo de buscar na literatura o que é e quais os desafios da vigilância em saúde do trabalho. Muitos desafios precisam ser enfrentados, tais como poucas ações de vigilância, fraca articulação intrassetorial, inexistência de pactos interssetoriais. Faz-se necessário que as equipes da ESF/PSF sejam sensibilizadas e capacitadas continuadamente para desenvolver e assumir as praticas necessárias.

Palavras-chave: vigilância - saúde do trabalhador - desafios.

\begin{abstract}
The Vigilance in Worker Health works in the relations of the work process with health and is a fundamental means to promote the health of workers and the prevention of accidents and workrelated diseases; Being essential to reflect on concepts and policies aimed at the health of the worker. This article has a qualitative approach of descriptive bibliographic type, so this review has the objective of searching in the literature what it is and what the challenges of surveillance in occupational health. Many challenges need to be addressed, such as few surveillance actions, weak intrinsetorial articulation, non-intersectoral pacts. ESF/PSF teams need to be sensitized and continuously trained to develop and take the necessary practices.
\end{abstract}

Key words: surveillance - worker health - challenges.

\section{INTRODUÇÃO}

A Vigilância em Saúde do Trabalhador atua nas relações do processo de trabalho com a saúde e é um meio fundamental para promover a saúde dos trabalhadores e a prevenção

\footnotetext{
${ }^{1}$ Fisioterapeuta. Especialista em Fisioterapia do Trabalho e Ergonomia pela Faculdade Unyleya. Mestre em Ciências Ambientais e Saúde pela Pontifícia Universidade Católica de Goiás - PUC Goiás - Email: geysefisioterapia@gmail.com

Revista Labor Fortaleza/CE, jan/jul 2017 Vol. 01, nº 18, p. 50-60 ISSN 1983-5000
} 
de acidentes e de doenças relacionadas ao trabalho; sendo imprescindível refletir sobre conceitos e políticas voltadas para a saúde do trabalhador.

Vários são os fatores que levam ao adoecimento e a acidentes de trabalho.

\begin{abstract}
As elevadas cargas e os processos de trabalho insalubres e perigosos, os equipamentos e as tecnologias ultrapassadas, os ambientes de trabalho inóspitos, as novas formas de organização e divisão do trabalho, a flexibilidade nos contratos e a consequente perda de direitos de proteção ou garantias, entre vários fatores, aumentam o adoecimento, a invalidez e a exclusão dos trabalhadores do mercado de trabalho (ANTUNES, 1995; MINAYO-GOMEZ; THEDIM-COSTA, 1997; ALVES, 2000; ABREU; RESENDE, 2001; TOLDRA et al., 2010 apud DALDON e LANCMAN, 2013, p. 93).
\end{abstract}

As ações de saúde do trabalhador são recentes, no Brasil, a expressão Saúde do Trabalhador (ST) começou nos anos 70 e 80 do século passado indicando o campo de conhecimentos vinculado a uma área ou programa de saúde (DALDON e LANCMAN, 2013).

Quanto à implantação de ações de vigilância em saúde do trabalhador, esta desencadeada por grupos institucionais de vários pontos do Brasil, foram observadas diferenças regionais que se relacionam com a força e qualidade da organização dos trabalhadores quanto às questões de saúde e, em termos institucionais, dependem das políticas regionais e da estrutura organizacional, da capacidade instalada, da qualidade dos profissionais envolvidos e de influências. Porem o tema comum a todos pode ser generalizado pela definição de vigilância em saúde do trabalhador e corresponde a um processo de articulação de ações de controle sanitário no sentido de promoção, proteção e atenção à saúde dos trabalhadores. Esse tema surge de dois campos de atuação da saúde pública, a vigilância em saúde e a saúde do trabalhador (MACHADO, 2003).

As exigências de um mundo contemporâneo nas relações de trabalho deixam claro que é preciso compreender melhor o papel exercido pelo trabalho na determinação do processo saúde-doença. Os modelos tradicionais não atendem aos novos paradigmas na dinâmica que aí está, de um mundo diversificado, globalizado. Sendo necessária a investigação constante, análise e intervenção num processo continuo de avaliação.

O conhecimento dos riscos e danos à saúde relacionados com o trabalho é fundamental para o planejamento das ações de assistência, de vigilância e de intervenção sobre os ambientes de trabalho, como também para a conduta dos trabalhadores e da sociedade. Para atuar com vigilância em saúde do trabalhador é imprescindível que saibamos conceitos e ações 
que permitam fluência e direções a serem seguidas. Visando, assim, garantir a qualidade na promoção e prevenção de saúde, notificação e tratamento da doença.

Dessa forma esta revisão tem o objetivo de buscar na literatura o que é e quais os desafios da vigilância em saúde do trabalho, assim descrever conceitos e reflexões sobre a vigilância em saúde do trabalhador no Brasil.

\section{METODOLOGIA}

A pesquisa terá uma abordagem qualitativa do tipo bibliográfica descritiva. Pretende descrever sobre a vigilância em saúde do trabalhador a partir de um levantamento bibliográfico privilegiando dados qualitativos e quantitativos, tendo em vista que o assunto tem ligação direta com dados estatísticos e também de relações dialógicas. Assim será possível realizar um histórico sobre o tema, atualizar-se sobre ele, extrair problemas, contradições e supostas soluções.

Desta forma, a revisão bibliográfica é indispensável para obter uma ideia precisa sobre o estado atual dos conhecimentos sobre um tema, sobre suas lacunas e sobre a contribuição da investigação para o desenvolvimento do conhecimento (LAKATOS E MARCONI, 2010). E a descrição é para Cervo, Bervian e da Silva (2007), um tipo de pesquisa que ocorre quando se registra, analisa e correlaciona fatos ou fenômenos, sem manipulá-los.

Para realizar a discussão a abordagem qualitativa permite uma maior liberdade teórico-metodológica utilizando, basicamente o método indutivo, que parte se situações particulares para generalizações (QUERINO et al, 2016).

As etapas da elaboração da presente revisão foram as seguintes: estabelecimento da hipótese e objetivos da revisão; estabelecimento de critérios de inclusão e exclusão de artigos (seleção da amostra); definição das informações a serem extraídas dos artigos selecionados; análise dos resultados e discussão.

A questão norteadora desta revisão foi: Qual o conceito e os desafios da vigilância em saúde do trabalhador no Brasil? Foram incluídos artigos no período compreendido entre 2012 a 2016, publicados em português com os textos completos disponíveis nas bases de dados selecionadas. Serão excluídos os artigos que, apesar de trazer os critérios de inclusão, acima descritos, não atendam ao objetivo do estudo. As palavras-chave utilizadas foram: vigilância, saúde do trabalhador e desafios; através de busca pelo acesso on-line na base de dados CAPES, utilizando os critérios de inclusão.

Revista Labor Fortaleza/CE, jan/jul 2017 Vol. 01, nº 18, p. 50-60 ISSN 1983-5000 


\section{RESULTADOS}

Nesta revisão foram encontrados 23 artigos que após o descarte dos repetidos, das revisões, dos indisponíveis, ou dos que não atendem ao objetivo a amostra final foi constituída de 7 artigos, conforme Quadro 1. Quanto ao tipo de pesquisa houve variados estudos e métodos dentro da pesquisa qualitativa e quantitativa. No que se refere aos periódicos dos artigos selecionados foram identificadas as áreas de saúde coletiva e saúde ocupacional. Todos os artigos se tratavam de Vigilância em saúde do trabalhador e se relacionaram com a Atenção Básica, a Atenção Primária, a Estratégia de Saúde da Família e com o SUS, se tratando principalmente sobre os desafios.

Quadro 1 - Artigos da amostra final.

\begin{tabular}{|c|c|c|c|}
\hline $\begin{array}{c}\text { Artig } \\
\mathbf{0}\end{array}$ & Autores & Título & $\begin{array}{l}\text { Periódico e ano de } \\
\text { publicação }\end{array}$ \\
\hline 1 & $\begin{array}{l}\text { DIAS, E C; } \\
\text { SILVA, T L; } \\
\text { ALMEIDA; } \\
\text { M H C }\end{array}$ & $\begin{array}{l}\text { Desafios para a construção cotidiana } \\
\text { da Vigilância em Saúde Ambiental e } \\
\text { em Saúde do Trabalhador na Atenção } \\
\text { Primária à Saúde }\end{array}$ & $\begin{array}{l}\text { Cad. Saúde Colet., } \\
\text { 2012, Rio de Janeiro, } \\
20 \text { (1): } 15-24\end{array}$ \\
\hline 2 & $\begin{array}{l}\text { LEAO, L H } \\
\text { C; BRANT, } \\
\quad \text { L C }\end{array}$ & $\begin{array}{l}\text { Manifestações de sofrimento: dilemas } \\
\text { e desafios para a vigilância em saúde } \\
\text { do trabalhador }\end{array}$ & $\begin{array}{l}\text { Revista de Saúde } \\
\text { Coletiva, Rio de } \\
\text { Janeiro, 25 [ 4 ]: } \\
\text { 1271-1292, 2015 }\end{array}$ \\
\hline 3 & $\begin{array}{l}\text { SOUZA, T S; } \\
\text { VIRGENS, L } \\
\text { S }\end{array}$ & $\begin{array}{l}\text { Saúde do trabalhador na Atenção } \\
\text { Básica: interfaces e desafios }\end{array}$ & $\begin{array}{c}\text { Rev. bras. Saúde } \\
\text { ocup., São Paulo, } 38 \\
\text { (128): 292-301, } 2013 \\
\end{array}$ \\
\hline 4 & $\begin{array}{l}\text { COSTA, D et } \\
\text { al }\end{array}$ & $\begin{array}{l}\text { Saúde do Trabalhador no SUS: } \\
\text { desafios para uma política pública }\end{array}$ & $\begin{array}{c}\text { Rev. bras. Saúde } \\
\text { ocup., São Paulo, } 38 \\
\text { (127): } 11-30,2013\end{array}$ \\
\hline 5 & $\begin{array}{l}\text { VASCONCE } \\
\text { LOS, L C F; } \\
\text { GOMES, C } \\
\text { M; } \\
\text { MACHADO, } \\
\text { J M H }\end{array}$ & $\begin{array}{l}\text { Entre o definido e o por fazer na } \\
\text { Vigilância em Saúde do trabalhador }\end{array}$ & $\begin{array}{l}\text { Ciência \& Saúde } \\
\text { Coletiva, } \\
\text { 19(12):4617-4626, } \\
2014\end{array}$ \\
\hline 6 & $\begin{array}{l}\text { LEÃO L H } \\
\text { C, CASTRO } \\
\text { A C. }\end{array}$ & $\begin{array}{l}\text { Políticas públicas de saúde do } \\
\text { trabalhador: análise da implantação de } \\
\text { dispositivos de institucionalização em } \\
\text { uma cidade brasileira } \\
\end{array}$ & $\begin{array}{c}\text { Ciência \& Saúde } \\
\text { Coletiva, 18(3):769- } \\
778,2013\end{array}$ \\
\hline 7 & $\begin{array}{l}\text { LACAZ, } \\
\text { F.A.C. et al }\end{array}$ & $\begin{array}{l}\text { Estratégia Saúde da Família e Saúde } \\
\text { do Trabalhador: um diálogo possível? }\end{array}$ & $\begin{array}{l}\text { Interface - Comunic., } \\
\text { Saude, Educ., v.17, }\end{array}$ \\
\hline
\end{tabular}




\begin{tabular}{|l|l|l|l|}
\hline & & n.44, p.75-87, \\
& & jan./mar. 2013 \\
\hline
\end{tabular}

\section{DISCUSSÃO}

Foi possível observar que as diferenças entre os textos estavam em seus objetivos.

O Quadro 2 apresenta esses objetivos e os resultados e conclusões atingidos. Porém o objetivo deste estudo é de conceituar Vigilância em Saúde do Trabalhador e descrever seus desafios, assim seguem as categorias.

Quadro 2 - Objetivos, resultados e conclusões dos artigos.

\begin{tabular}{|c|c|c|}
\hline Artigo & Objetivo & Resultados e conclusões \\
\hline 1 & $\begin{array}{l}\text { Apresentar aspectos conceituais } \\
\text { e políticos institucionais que } \\
\text { aproximam os campos da Saúde } \\
\text { do Trabalhador e Saúde } \\
\text { Ambiental, e discutir as } \\
\text { possibilidades e os desafios para } \\
\text { a construção cotidiana de ações } \\
\text { de Vigilância em Saúde do } \\
\text { Trabalhador e Saúde Ambiental } \\
\text { nas práticas da Atenção } \\
\text { Primária, tendo como base os } \\
\text { processos produtivos instalados } \\
\text { no território das equipes }\end{array}$ & $\begin{array}{l}\text { Para que ocorra a incorporação dessas ações } \\
\text { na rede de Atenção Primaria devem ser } \\
\text { implementadas mudanças no processo de } \\
\text { trabalho das equipes para a efetivação da } \\
\text { proposta e preparação da rede a fim de } \\
\text { responder às questões de Saúde Ambiental e } \\
\text { Saúde do Trabalhador: estruturar a linha de } \\
\text { cuidado, reforçar a integração com outros } \\
\text { setores e atores (sindicatos, associações de } \\
\text { moradores, meio ambiente), entre outras. }\end{array}$ \\
\hline 2 & $\begin{array}{l}\text { Realizar uma análise crítica dos } \\
\text { desafios e dilemas relativos à } \\
\text { integração da saúde mental no } \\
\text { escopo da vigilância em saúde } \\
\text { do trabalhador no Sistema Único } \\
\text { de Saúde }\end{array}$ & $\begin{array}{l}\text { Para avançar, faz-se necessário enfrentar o } \\
\text { desafio de compreender os fenômenos em } \\
\text { saúde mental e de lidar com situações } \\
\text { complexas, dadas as múltiplas manifestações } \\
\text { do sofrimento relacionado ao trabalho e suas } \\
\text { diferenças e níveis. Isso implica } \\
\text { problematizar os dilemas para a produção de } \\
\text { informação e as práticas de notificação no } \\
\text { Sinan. }\end{array}$ \\
\hline 3 & $\begin{array}{l}\text { Refletir acerca das interfaces da } \\
\text { Saúde do Trabalhador (ST) na } \\
\text { Atenção Básica }(\mathrm{AB}) \text { do sistema } \\
\text { de saúde brasileiro }\end{array}$ & $\begin{array}{l}\text { As categorias de análise possibilitaram } \\
\text { estabelecer o resgate histórico da ST no SUS } \\
\text { e refletir acerca de elementos da ST na AB, } \\
\text { como: território e ferramentas de atenção à } \\
\text { saúde; competência das equipes da atenção } \\
\text { básica; vigilância em saúde do trabalhador; } \\
\text { relações de produção e saúde ambiental; } \\
\text { práticas educativas em ST; vulnerabilidades } \\
\text { do trabalhador da saúde e implementação de } \\
\text { experiências institucionais. A incorporação }\end{array}$ \\
\hline
\end{tabular}

Revista Labor Fortaleza/CE, jan/jul 2017 Vol. 01, nº 18, p. 50-60 ISSN 1983-5000 


\begin{tabular}{|c|c|c|}
\hline & & $\begin{array}{l}\text { da ST na AB caracteriza-se como elemento } \\
\text { para redefinição de práticas e mudança no } \\
\text { modelo assistencial. }\end{array}$ \\
\hline 4 & $\begin{array}{l}\text { Fomentar a discussão, o } \\
\text { entendimento, a implementação } \\
\text { e o avanço das ações em Saúde } \\
\text { do Trabalhador, que deveriam } \\
\text { estar no centro das políticas } \\
\text { públicas. }\end{array}$ & $\begin{array}{l}\text { As atitudes de ST pressupõem, por sua } \\
\text { natureza, além de recursos materiais, quadro } \\
\text { de profissionais satisfatório capacitado e } \\
\text { dotado de carreira compatível com as } \\
\text { atividades primordiais do Estado provedor, } \\
\text { condição mínima para o confronto dos } \\
\text { problemas complexos do campo da ST, ou } \\
\text { seja, uma política de Estado condizente com } \\
\text { a perspectiva de superação da precariedade do } \\
\text { trabalho contemporâneo e com a proteção } \\
\text { integral da saúde dos trabalhadores. }\end{array}$ \\
\hline 5 & $\begin{array}{l}\text { Trazer algumas indagações } \\
\text { sobre a Vigilância em Saúde do } \\
\text { Trabalhador(VISAT), no Brasil, } \\
\text { a partir de vários elementos de } \\
\text { análise, visando subsidiar o } \\
\text { debate sobre o tema na IV } \\
\text { Conferência Nacional de Saúde } \\
\text { do Trabalhador e da } \\
\text { Trabalhadora. }\end{array}$ & $\begin{array}{l}\text { Conclui-se que é preciso intenso investimento } \\
\text { na formação de indivíduos e na composição } \\
\text { de normas específicas para executar ações } \\
\text { sistemáticas e intersetoriais a esse respeito. } \\
\text { No momento de realização da Conferência, a } \\
\text { maior expressão do exercício do controle } \\
\text { social, é aconselhável que se avalie a sua } \\
\text { atuação nos processos de vigilância, enquanto } \\
\text { pressuposto para garantir a eficácia dessas } \\
\text { estratégias. }\end{array}$ \\
\hline 6 & $\begin{array}{l}\text { Refletir sobre a omissão de } \\
\text { práticas voltadas para a saúde } \\
\text { dos trabalhadores no âmbito de } \\
\text { atuação da Estratégia Saúde da } \\
\text { Família (ESF) e propor a } \\
\text { inclusão dessas práticas. }\end{array}$ & $\begin{array}{l}\text { Há uma lacuna a ser preenchida pela prática } \\
\text { da ESF e uma possibilidade de } \\
\text { relacionamento da ESF com a ST deve } \\
\text { considerar o trabalho em domicílio e seus } \\
\text { desdobramentos, como o trabalho do menor e } \\
\text { da mulher. E preciso repensar o } \\
\text { monitoramento dos locais de trabalho que } \\
\text { fazem parte da vida social na área de } \\
\text { abrangência de uma Unidade Básica de } \\
\text { Saúde. }\end{array}$ \\
\hline 7 & $\begin{array}{l}\text { Analisar sob o horizonte mais } \\
\text { amplo da história dos Programas } \\
\text { de Saúde do Trabalhador no } \\
\text { Brasil e do surgimento da } \\
\text { Renast, a implantação do Cerest } \\
\text { de acordo com a especificidade } \\
\text { de suas relações de proveniência } \\
\text { e emergência, conforme } \\
\text { Foucault, fazendo um resgate da } \\
\text { trajetória do Programa de Saúde } \\
\text { do Trabalhador do referido } \\
\text { município. }\end{array}$ & $\begin{array}{l}\text { Os resultados permitem dizer que o Cerest } \\
\text { possui uma história de isolamento e } \\
\text { marginalidade na estrutura política e } \\
\text { institucional da região, atua continuamente } \\
\text { numa lógica predominantemente assistencial } \\
\text { desde sua criação, e enfrenta sérios entraves } \\
\text { políticos, ao longo do tempo, na } \\
\text { implementação das ações de saúde do } \\
\text { trabalhador. }\end{array}$ \\
\hline
\end{tabular}

Conceitos apresentados

Revista Labor Fortaleza/CE, jan/jul 2017 Vol. 01, nº 18, p. 50-60 
Nem todos os artigos conceituaram a Vigilância em Saúde do Trabalho, mas alguns trazem que a Vigilância da Saúde do Trabalhador se enquadra no Ministério da Saúde dentro da Vigilância em Saúde e, portanto, dentro da Atenção primária e citam a Portaria no 3.252 de 22 de Dezembro de 2009 que diz que a Vigilância da Saúde do Trabalhador visa à promoção da saúde e à redução da morbimortalidade da população trabalhadora, por meio da integração de ações que intervenham nos agravos e seus determinantes decorrentes dos modelos de desenvolvimento e processo produtivos (DIAS, SILVA e ALMEIDA, 2012; COSTA et al, 2013; VASCONCELOS, GOMES e MACHADO, 2013; LEÃO e CASTRO, 2013).

A Saúde do Trabalhador é caracterizada como campo de práticas e conhecimentos que buscam conhecer e intervir nas relações de trabalho e saúde-doença. Ela ainda pode ser entendida enquanto prática teórica interdisciplinar e prática político-ideológica (superação das relações de poder e conscientização dos trabalhadores), desenvolvidas por diversos atores situados em lugares sociais distintos e informados por uma perspectiva comum (SOUZA e VIRGENS, 2013). Esse artigo traz a definição pelo Ministério da Saúde como:

\footnotetext{
Uma atuação contínua e sistemática, ao longo do tempo, no sentido de detectar, conhecer, pesquisar e analisar os fatores determinantes e condicionantes dos agravos à saúde, relacionados aos processos e ambientes de trabalho, em seus aspectos tecnológico, social, organizacional e epidemiológico, com a finalidade de planejar, executar e avaliar intervenções sobre esses aspectos, de forma a eliminá-los e controlá-los (BRASIL, 1998, Anexo I apud SOUZA e VIRGENS, 2013).
}

Os conceitos se corroboram, postos que estes vêem de base legislativa como a lei Orgânica da Saúde (Lei 8080/199017, que inclui ações em saúde do trabalhador), e a proposta do Ministério da Saúde que privilegia a organização dessas ações em Centros de Referência em Saúde do Trabalhador (Cerest) e a Rede Nacional de Atenção Integral à Saúde do Trabalhador (Renast).

Desafios

Apesar de limitadas e parciais já existem ações de Vigilância em Saúde do Trabalho, porém muitos desafios precisam ser enfrentados. Nesse âmbito, embora uma parcela significativa de usuários atendidos seja de trabalhadores, o reconhecimento do trabalho na 
determinação dos processos saúde-doença traduzido na orientação das intervenções permanece marginal ou inexistente (DIAS, SILVA e ALMEIDA, 2012).

Ainda no estudo de Dias, Silva e Almeida (2012) trabalhos revelaram que, em muitos municípios, as equipes da Atenção Primária a Saúde (APS) estão preocupadas com as relações entre trabalho-saúde-doença e ambiente, desenvolvendo estratégias de intervenção, embora muitas vezes essas ações não fizessem parte do cotidiano das equipes. Também predominaram atividades de capacitação dos profissionais da APS, principalmente com base nos Protocolos de Complexidade Diferenciada dos Agravos Relacionados ao Trabalho. A ênfase desses processos foi dada no desenvolvimento de ações assistenciais, de diagnóstico e notificação, ficando as ações de promoção e vigilância em segundo plano. Os Agentes Comunitários de Saúde (ACS) também se queixaram da falta de preparo técnico e de apoio institucional para lidar com essas questões.

Esses autores também trazem as relações fortes que o trabalho tem com o meio ambiente e estes com todos os setores, portanto a vigilância em saúde deve ser intersetorial. Eles sugerem que devem ser implementadas mudanças no processo de trabalho das equipes para a efetivação da proposta e preparação da rede a fim de responder às questões de Saúde Ambiental e Saúde do Trabalhador: estruturar a linha de cuidado, reforçar a integração com outros setores e atores (sindicatos, associações de moradores, meio ambiente), entre outras (DIAS, SILVA e ALMEIDA, 2012).

A efetividade das ações de Vigilância em Saúde do Trabalhador (VISAT) esbarra em entraves institucionais e políticos, como a insuficiente capacitação de profissionais, pouco apoio financeiro, material e técnico para as atuações in loco, baixo engajamento dos sindicatos e representações de trabalhadores junto aos serviços de saúde e os conflitos com setores empresariais e estatais. A fragmentação das vigilâncias sanitária, ambiental, epidemiológica para as intervenções nos processos de produção, somada à ênfase assistencial do Sistema Único de Saúde (SUS), também é um fator que dificulta a implementação das práticas (LEÃO e BRANT, 2015).

Conforme o artigo que trata da saúde mental do trabalhador, uma das questões mais urgentes a ser enfrentada pela VISAT é o sofrimento dos trabalhadores relativas aos problemas psíquicos, assédios, estresse e outras manifestações. Isso decorre do atual modo de produçãoconsumo e das características técnicas e sociais da organização pós-fordista, que induzem a novos sofrimentos (LEÃO e BRANT, 2015).

Revista Labor Fortaleza/CE, jan/jul 2017 Vol. 01, nº 18, p. 50-60 ISSN 1983-5000 
Apesar dos avanços conquistados, ainda existem muitos desafios como: o combate ao reducionismo das políticas públicas, os entraves políticos no âmbito municipal, e o desconhecimento de muitos gestores; considerar com responsabilidade o trabalho infantil, o informal, o trabalho de rua e o desemprego; a expansão da atuação para o setor de serviços e agricultura. Faz-se necessário, ainda, a ampliação dos estudos e a reformulação de referenciais conceituais e metodológicos; uma formação acadêmica que reconheça a relação entre capital e trabalho; a capacitação dos profissionais da saúde para lidar com os riscos e os agravos relacionados ao trabalho; e movimentos sociais e sindicais atuantes (SOUZA e VIRGENS, 2013).

As próprias leis exprimem dubiedade e fragilidade das ações de vigilância, na ausência de prioridades e programas articulados nos vários níveis do território (COSTA et al, 2013). A atuação no Controle Social, na própria estrutura do SUS, apresenta enormes as dificuldades de apoio para a VISAT, sobretudo por parte da gestão nos seus variados níveis e em instâncias intrassetoriais em que haja responsabilidades diretas ou indiretas sobre estas questões (VASCONCELOS, GOMES e MACHADO, 2013).

Parece que a rede SUS ainda não integrou em suas concepções, paradigmas e atuações, o espaço que o Trabalho abrange na vida dos indivíduos e suas relações com o espaço sócio-ambiental, ainda que a proposta da Renast procura fornecer auxílio técnico e científico às intervenções em ST em toda rede do SUS, até mesmo na Atenção Básica (LACAZ et al, 2013).

A questão da rotatividade das equipes, com relações celetistas, em particular dos médicos e psicólogos, e a frágil relação com o movimento sindical são as maiores dificuldades para o desenvolvimento de educação continuada na Atenção Básica (LACAZ et al, 2013). Poucas ações de vigilância, fraca articulação intrassetorial, inexistência de pactos interssetoriais, baixa comunicação em saúde do trabalhador entre os municípios da região, entre outras coisas (LEAO e CASTRO, 2013).

\section{CONSIDERACOES FINAIS}

As ações de saúde da ESF, referenciadas no território sob a responsabilidade da equipe, permitem o reconhecimento e/ou a identificação de situações de risco ou perigos para a saúde, originários nos processos produtivos e em situações de trabalho, facilitando a vigilância e o provimento de assistência adequada às necessidades daquela população, particularmente no 
contexto das mudanças nas configurações de trabalho, o crescimento do mercado informal, domiciliar e familiar.

Porem compreende-se que há uma grande lacuna a ser preenchida pela prática da Estratégia Saúde da Família e que uma possibilidade de relação com a ST precisa também levar em conta o trabalho em domicílio, o trabalho infantil e da mulher. Entretanto não só, é preciso repensar o controle dos ambientes de trabalho que fazem parte da vida social na área de abrangência de uma UBS, dessa forma é necessário considerar a necessidade de elaboração de estratégias de operacionalização que possam ser incluídas na prática do dia a dia das equipes da ESF.

Diante do exposto é necessário que as equipes da ESF/PSF sejam sensibilizadas e qualificadas continuadamente para desenvolver e apropriar tais práticas, o que constitui tanto um desafio como uma superação dos limites de sua atuação, quando esta deixa de lado um elevado contingente populacional que atua no espaço privilegiado de sua intervenção.

\section{REFERÊNCIAS}

CERVO, Amado Luiz; BERVIAN, Pedro Alcino; DA SILVA, Roberto. Metodologia Científica. 6. ed. São Paulo: Pearson Prentice Hall, 2007.

COSTA, D et al. Saúde do Trabalhador no SUS: desafios para uma política pública. Rev. bras. Saúde ocup., São Paulo, 38 (127): 11-30, 2013

DALDON, Maria Tereza Bruni; LANCMAN, Selma. Processo em saúde do trabalhador rumos e incertezas. Rev. bras. saúde ocup. vol.38 no.127 São Paulo Jan./June 2013

DIAS, E C; SILVA, T L; ALMEIDA, M H C. Desafios para a construção cotidiana da Vigilância em Saúde Ambiental e em Saúde do Trabalhador na Atenção Primária à Saúde . Cad. Saúde Colet., 2012, Rio de Janeiro, 20 (1): 15-24

LACAZ, F.A.C. et al. Family Estratégia Saúde da Família e Saúde do Trabalhador: um diálogo possível? Interface - Comunic., Saude, Educ., v.17, n.44, p.75-87, jan./mar. 2013

LAKATOS, E. Maria; MARCONI, M. de Andrade. Fundamentos de metodologia científica: Técnicas de pesquisa. 7 ed. - São Paulo: Atlas, 2010.

LEÃO, L H C; BRANT, L C. Manifestações de sofrimento: dilemas e desafios para a vigilância em saúde do trabalhador. Revista de Saúde Coletiva, Rio de Janeiro, 25 [ 4 ]: 1271-1292, 2015 
LEÃO L H C, CASTRO A C. Políticas públicas de saúde do trabalhador: análise da implantação de dispositivos de institucionalização em uma cidade brasileira. Ciência $\boldsymbol{\&}$ Saúde Coletiva, 18(3):769-778, 2013

MACHADO, Jorge Mesquita Huet. Processo de vigilância em saúde do trabalhador. Cad.

Saúde Pública, Rio de Janeiro, 19(1):319-322, jan-fev, 2003

QUERINO et al. Metodologia da Pesquisa e da Produção Científica. Brasília: 2016

SOUZA, T S; VIRGENS, L S. Saúde do trabalhador na Atenção Básica: interfaces e desafios. Rev. bras. Saúde ocup., São Paulo, 38 (128): 292-301, 2013.

VASCONCELOS, L C F; GOMES, C M; MACHADO, J M H. Entre o definido e o por fazer na Vigilância em Saúde do trabalhador. Ciência \& Saúde Coletiva, 19(12):4617-4626, 2014. 